\title{
Résister à la calomnie : Changeling de Clint Eastwood
}

Roberta Martina Zagarella

\section{(2) OpenEdition \\ 12 Journals}

Édition électronique

URL : http://journals.openedition.org/rhetorique/407

DOI : $10.4000 /$ rhetorique.407

ISSN : 2270-6909

Éditeur

UGA Éditions/Université Grenoble Alpes

Édition imprimée

ISBN : 978-284310-303-2

Référence électronique

Roberta Martina Zagarella, «Résister à la calomnie : Changeling de Clint Eastwood », Exercices de rhétorique [En ligne], 5 | 2015, mis en ligne le 15 septembre 2015, consulté le 12 septembre 2020. URL : http://journals.openedition.org/rhetorique/407 ; DOI : https://doi.org/10.4000/rhetorique.407

Ce document a été généré automatiquement le 12 septembre 2020.

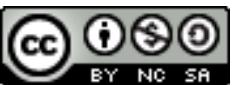

Les contenus de la revue Exercices de rhétorique sont mis à disposition selon les termes de la Licence Creative Commons Attribution - Pas d'Utilisation Commerciale - Partage dans les Mêmes Conditions 4.0 International. 


\title{
Résister à la calomnie : Changeling de Clint Eastwood
}

\author{
Roberta Martina Zagarella
}

\author{
We find, then, that when the individual is in the \\ immediate presence of others, his activity will have a \\ promissory character. \\ On constate que lorsque quelqu'un est en \\ présence d'autrui, son activité a toutes les \\ caractéristiques d'un engagement à terme ${ }^{1}$.
}

\section{1. « Je vous le jure. C'est votre enfant »}

1 Est-il jamais possible qu'une mère ne reconnaisse plus son propre enfant? Quel est le prix de la certitude maternelle et de la confiance en ce qu'elle ressent?

2 Nous sommes en 1928, à Los Angeles. Dans Changeling, Clint Eastwood met en scène un drame en nous racontant une histoire tirée d'un fait qui a réellement eu lieu ${ }^{2}$. Christine Collins (Angelina Jolie) est une mère célibataire, chef de service d'un centre d'appel téléphonique. Un samedi matin, obligée de se rendre au travail pour le remplacement d'un collègue, elle laisse son enfant âgé de neuf ans, tout seul à la maison. À son retour, l'enfant a disparu. Cinq mois après, le département de la police semble avoir réglé l'affaire. Christine se précipite à la gare pour embrasser à nouveau son fils, sauf qu'elle ne reconnaît pas l'enfant qui descend du train. Immobile devant ce petit garçon, elle s'adresse au capitaine de la Police J. J. Jones (Jeffrey Donovan), en lui disant : « Ce n'est pas mon fils. " Percevant le trouble de la mère, le capitaine essaie de la rassurer face à l'enfant, visiblement amaigri et changé par ces mois épouvantables. De surcroît, dès qu'on l'interpelle, le petit garçon confirme la version de l'officier de police, en affirmant être Walter Collins, c'est-à-dire le fils de Christine.

3 La mère de l'enfant, bien qu'elle soit sûre que le garçon n'est pas du tout Walter, s'attarde, doute d'elle-même et fait confiance au capitaine ; de son côté, celui-ci arrive à 
la persuader qu'elle est dans un état de confusion et qu'elle doit ramener son fils à la maison:

Capitaine J. J. Jones. - Je vous le jure. Je vous le promets. Je vous en donne ma parole. C'est votre enfant. Si vous avez un problème, n'hésitez pas à m'en parler. Je m'en occuperai. Je vous donne ma parole. Faites-moi confiance [...].

Christine Collins. - Je n'ai peut-être pas les idées claires. Et... D'accord.

Capitaine J. J. Jones. - Vous n'avez pas les idées claires, c'est exactement ça.

Le "drame dans le drame » démarre avec ce serment, ou mieux, avec la confiance que Christine a dans le "sacrement du pouvoir ${ }^{3}$ " : c'est son obstination à affirmer que la police s'est trompée qui amènera Christine à être d'abord dépeinte comme une personne instable émotionnellement et à être par conséquent qualifiée comme une mère se dérobant vis-à-vis de ses responsabilités. Ensuite, elle sera présentée comme paranoïaque, affectée par des délires de persécution et détachée de la réalité. Jugée comme un danger pour elle-même et les autres, elle sera internée dans l'asile psychiatrique du Los Angeles County General Hospital.

5 La structure narrative du film se déroule dans son intégralité à partir de cet épisode, et plus particulièrement à partir de l'hésitation de Christine à la gare. Dans ce conflit entre deux points de vue divergents - celui de Christine et celui du capitaine de police - le doute de la femme devient le pivot autour duquel le rapport entre discours et vérité s'articule. Nous allons constater que l'argumentation diffamatoire de la police et du psychiatre portera sur ce genre de questionnement : si cette femme était tellement sûre que l'enfant n'était pas Walter, pourquoi aurait-elle ramené à la maison un enfant qui n'était pas son fils?

6 Avant d'entrer dans les détails des stratégies utilisées par Christine Collins pour résister aux calomnies et se défendre de l'opprobre jeté sur elle par une police corrompue, il est intéressant de souligner les motivations poussant la femme à douter d'elle-même, malgré sa certitude maternelle. Le film présente l'avantage de nous montrer l'articulation du rapport entre crédibilité et discours à l'intérieur de deux situations opposées: Christine a tendance à faire confiance au capitaine par le biais de l'ethos que celui-ci manifeste par son comportement et ses mots; tandis que les autorités refusent d'admettre qu'ils aient pu commettre une erreur et font ainsi appel à l'image sociale de la femme pour rendre vaines ses protestations.

7 Pourquoi Christine se laisse-t-elle persuader par le capitaine? Pourquoi croyons-nous en ce que nous dit un inconnu, plutôt que d'avoir confiance en nous-mêmes? Le rôle institutionnel que l'homme incarne, ainsi que l'état de trouble et confusion de Christine, constituent certainement deux raisons qui poussent Christine à faire confiance au capitaine Jones. Mais ces motivations ne sont pas les seules raisons. En effet, c'est le discours du capitaine qui inspire la confiance de Christine en le rendant fiable à ses yeux; la crédibilité de l'officier de police émane des mots qu'il prononce, et en particulier de l'acte linguistique qui, par antonomase, a le pouvoir de lier foi et parole: le serment.

8 Le besoin du serment trouve son origine dans des situations critiques où la fides est brisée ou affaiblie; en même temps, la force des mots « je jure » réside effectivement dans la fides, c'est-à-dire dans la relation réciproque que les sujets parlants établissent, l'un accordant sa propre confiance et l'autre jouissant de la confiance de l'autre ${ }^{4}$.

9 Il ne faut pas oublier que, d'un point de vue linguistique, le serment est considéré comme un énoncé performatif, faisant partie d'une catégorie d'actes langagiers dont 
l'énonciation coïncide avec une véritable action. Selon les théories, notamment celles d'Austin ${ }^{5}$ et de Benveniste ${ }^{6}$, il y a une différence entre les énoncés «il jure » et «je jure » : tandis que l'énoncé «il jure » décrit un état des choses, en prononçant les mots «je jure », on réalise et on accomplit effectivement l'action de jurer, qui disparaît en l'absence de l'énonciation même. Contrairement aux autres êtres vivants, par un serment, l'être humain a donc la possibilité de se mettre en jeu à l'intérieur même de son discours, de s'engager à rendre compte de ses mots par sa propre vie, de témoigner de ce qu'il profère à la première personne. Le serment exprime l'exigence d'établir un lien à la fois éthique et politique entre les mots, les choses et les actions ${ }^{7}$. En énonçant la phrase " je vous jure que c'est votre enfant ", le capitaine Jones réalise un acte coactif de portée sociale, car le fait de donner sa propre parole marque la coïncidence entre vérité et discours du sceau de sa crédibilité. Le pouvoir d'obligation invoqué par le serment est tellement fort qu'il est à même de semer le doute chez Christine sur ses propres facultés de jugement.

10 Le performatif du capitaine, dès l'instant où il est prononcé, détermine les conséquences sociales et juridiques implicites en cas de parjure. Pour Jones, ces conséquences coïncideront avec la suspension définitive de l'exercice de ses fonctions. Plutôt que de se focaliser sur les caractéristiques définissant le serment, il est intéressant d'observer qu'il y a des situations où l'on est disposé à utiliser (et par conséquent à nous laisser persuader par) des verbes tels que "jurer ", "promettre ", parce qu'ils se révèlent parmi les plus puissants des dispositifs dont notre langue dispose pour indiquer la force illocutoire d'engagement ${ }^{8}$. Pour cette raison, on a souvent recours à des expressions comme «je jure» dans l'exécution d'actes linguistiques dans lesquels, bien qu'ils ne soient pas à proprement parler des serments, nous voulons souligner l'intensité de notre engagement pour gagner la confiance de notre interlocuteur. À ces occasions, le sujet se met en jeu et se contraint lui-même à la vérité de son affirmation: celui qui jure, en effet, "met en jeu quelque chose d'essentiel pour lui, une possession matérielle, sa parenté, même sa propre vie, pour garantir la véracité de son affirmation ${ }^{9}$ ». La fonction du serment ne réside donc pas «dans l'affirmation qu'il produit, mais dans la relation qu'il institue entre la parole prononcée et la puissance invoquée ${ }^{10} »$.

11 Ce qui émerge d'un serment (et en particulier s'il s'agit d'un serment assertif), c'est une modalité de se présenter aux autres en tant que garants de la vérité, dont la puissance réside dans la confiance que l'autre lui accorde, et dont l'enjeu est la facilitation d'une prise de décision dans des situations d'incertitude. De ce point de vue, la confiance constitue l'une des forces de synthèse les plus importantes au sein de la sociétée ${ }^{11}$, jouant un rôle décisif dans la praxis. Dans l'effort de faire un choix - au moment le plus opportun (kairos) - nous sommes poussés à chercher des épreuves, discursives ou non, nous permettant la meilleure délibération possible.

Incertaine sur le choix de ramener l'enfant à la maison avec elle ou non, Christine est obligée de choisir à cet instant, avec un nombre spécifique et très limité de connaissances à sa disposition. C'est ainsi qu'elle se laissera persuader et qu'elle se laissera berner par le discours du policier. 


\section{2. «Faites-moi confiance »}

13 Le serment et la promesse ne sont pas les seuls actes linguistiques se fondant sur la confiance. La possibilité même de se persuader mutuellement repose - d'un point de vue étymologique - sur une relation fiduciaire entre celui qui parle et celui qui écoute. Le mot pistis (substantif qui vient du grec peitho, je persuade) correspond, en effet, à la famille du latin fides: le verbe signifie "se laisser persuader ", mais aussi «avoir de la confiance ", « donner de l'écoute ", « obéir »; l'adjectif pistos et le substantif pistis, qui dérivent du verbe, signifient respectivement « digne de confiance », « fidèle », « loyal », " crédible», et " confiance", " foi », « croyance », " conviction", " persuasion ». Le mot pistis indique aussi bien la confiance qu'on accorde à quelqu'un que la crédibilité dont on profite et les garanties - objets ou discours - par laquelle la confiance été accordée ${ }^{12}$.

Le rappel sur la notion de pistis permet de préciser que la confiance et la crédibilité constituent des éléments techniques de l'argumentation rhétorique et révèle un deuxième aspect du discours du capitaine Jones, à savoir son autoreprésentation en tant que sujet parlant fiable («Faites-moi confiance»). Aristote, dans la Rhétorique, distingue trois types de preuves techniques (entechnoi pisteis ${ }^{13}$ ), qu'on peut obtenir par le discours : la preuve qui se fonde sur l'image de l'orateur (ethos), la preuve réalisée par l'auditeur (pathos) et la preuve qui réside dans le discours même ${ }^{14}(\log o s)$. La première preuve est étroitement liée à la confiance, car elle donne la mesure de la crédibilité que le sujet parlant réussit à construire par son propre discours. Pour Aristote, il ne faut pas penser que la fiabilité du locuteur ne contribue pas à la construction de son image persuasive. Au contraire, l'ethos constitue la plus forte des formes de pistis ${ }^{15}$. Pourtant, Aristote insiste sur un point : pour qu'on puisse nommer comme technique la preuve se réalisant par le caractère de l'orateur et induisant l'auditoire dans un état de croyance, l'ethos est obligé d'émerger par un discours prononcé et non pas par la réputation préalable de l'orateur ${ }^{16}$. Aristote ne nie pas qu'il existe une autorité émanant du charisme, de la réputation ou des discours précédents de l'orateur; cependant, il est plausible que pour lui ces éléments fassent partie d'un ethos non technique, c'est-à-dire - en se tenant à la définition - qui n'est pas construit dans le discours mais qui lui préexiste.

Le rapport entre ethos technique et non technique a fait l'objet de débats depuis l'Antiquité ; les études contemporaines y consacrent une attention particulière, surtout dans le domaine de la rhétorique, dans l'analyse du discours et dans la microsociologie développée à partir des enseignements de Goffman. Ainsi, la recherche est en train de se réorienter, par l'intégration de la preuve technique avec les aspects non techniques et même non verbaux, vers un élargissement de la notion d'ethos, impliquant l'ensemble des diverses modalités par lesquelles un sujet se présente dans les échanges conversationnels et discursifs, y compris ses déclinaisons quotidiennes et personnelles : habillement, style, gestes, voix, posture, appartenance à un groupe, rôle institutionnel, croyances implicites, pour n'en donner que quelques exemples ${ }^{17}$.

Christian Plantin suggère qu'il est possible de distinguer trois aspects de la subjectivité à l'intérieur de la notion contemporaine d'ethos ${ }^{18}$ :

1. Un aspect construit par le discours, aussi bien en tant que stratégie rhétorique qui affecte le sujet parlant qu'en tant que caractéristique intrinsèque de la langue ;

2. Un aspect qui correspond à une opinion préconçue sur le caractère de celui qui parle ; 
3. Un aspect construit par ce que l'orateur dit de lui-même, à savoir des éléments spécifiques d'autoreprésentation, thématisés consciemment par le locuteur afin de légitimer métonymiquement sa position. Ces éléments sont très différents de ce que l'orateur laisse deviner ou déduire indirectement sur lui-même.

17 Le troisième élément est fort controversé. En général on n'a pas confiance en quelqu'un parce que celui-ci affirme être quelqu'un de fiable ou parce qu'il désire qu'on lui fasse confiance. Au contraire, ce genre d'affirmations flatteuses sur soi-même peut irriter et conduire ceux qui écoutent à agir de façon totalement opposée. En effet, l'ethos a la propriété particulière de ne pas configurer un argument explicite. Il se montre dans le discours, sans pour autant constituer l'objet de ce discours particulier : il est l'image que l'orateur montre de soi-même en disant quelque chose d'autre.

Pourtant, dans certains cas il peut arriver que l'orateur dise quelque chose sur luimême, et cela constitue un élément explicite d'auto-narration. Ainsi, il peut contribuer positivement à la construction de l'image de l'orateur aux yeux de l'auditoire. Normalement, cela se vérifie - et certainement dans le cas que nous analysons: «Faites-moi confiance »-dans les cas où le contexte de l'ethos est connoté de façon décisive par la perception sociale des pratiques discursives et les relations de pouvoir. Ce n'est pas la même chose de se mettre en jeu en tant qu'homme public - et notamment en tant que capitaine de police - et de s'exposer à l'intérieur d'une conversation personnelle : le rôle de l'acteur social détermine la situation énonciative et le milieu établissant la confiance, en intervenant sur la nature persuasive de l'ethos. Aussi, l'orateur assume-t-il la fonction de contexte nécessaire à l'interprétation de l'acte linguistique ${ }^{19}$. L'énoncé «Faites-moi confiance», s'il est prononcé par deux locuteurs différents, ne sera pas retenu de la même façon et sera perçu sous une lumière différente.

19 Le sentiment de confiance se régule sur la base des modalités fixées par les sujets parlants et la société, définissant la situation de l'échange verbal. Eastwood nous offre deux situations opposées et complémentaires, en mettant en scène le rapport entre les attentes des rôles, le pouvoir et le langage. D'une part, nous avons vu que Christine a tendance à faire confiance au capitaine grâce à son rôle institutionnel, et d'autre part, les autorités corrompues exploitent la condition sociale de la femme pour rendre vaines ses protestations et ses déclarations, en abusant de leur pouvoir et en manipulant les preuves objectives et vérifiables. Dans le premier cas, le rôle du capitaine Jones contribue à renforcer la relation de confiance; en revanche, dans le deuxième, la situation de Christine devient à la fois un prétexte pour jeter le discrédit sur la vérité et l'authenticité de son discours, et un point d'appui pour justifier la décision brutale de modifier les données de la réalité en les déformant par une forme de resocialisation : l'internement non volontaire.

Nous allons à présent porter notre attention sur ce deuxième cas.

\section{L'épreuve de l'objectivité}

21 Rentrée chez elle, Christine s'aperçoit que, contrairement à Walter, le petit garçon que la police lui a rendu est circoncis. De plus, il est aussi plus petit que son fils de quelques centimètres. Persuadée et beaucoup plus sûre de sa première impression, elle décide de se présenter chez le capitaine Jones. Eastwood construit le dialogue des personnages 
autour de deux éléments-pivots : la mauvaise foi du département de la police et la caractérisation sociale de Christine Collins.

C. Collins. - Ce n'est pas mon fils!

J. J. Jones. - Pourquoi faites-vous ça, Mme Collins ? Pourquoi faites-vous ça ? Vous semblez être en mesure de prendre soin du garçon. Votre emploi paie assez pour subvenir à ses besoins. Pourquoi tentez-vous de fuir devant vos responsabilités maternelles?

C. Collins. - Je ne fuis devant rien! Je n'esquive pas mes responsabilités! Je m'occupe même de ce garçon, car il n'a personne d'autre que moi! Mais vous, vous ne cherchez plus mon fils!

J. J. Jones. - Pourquoi chercher quelqu'un qu'on a déjà trouvé ?

C. Collins. - Parce que vous ne l'avez pas trouvé.

J. J. Jones. - Son identité a été confirmée par les plus grands cerveaux du domaine de l'identification des enfants, par des gens qui savent ce qu'ils font.

C. Collins. - Et moi, je ne sais rien?

Après cet échange conversationnel, le docteur Tarr - spécialiste nommé par Jones - se rend chez Collins pour visiter l'enfant et rassurer Christine, en proie à un état d'anxiété maternelle. Du point de vue du docteur, tous les phénomènes observés après le retour de l'enfant sont susceptibles de faire l'objet d'une explication médicale parfaitement logique : un traumatisme pourrait avoir eu une influence sur la croissance des enfants, le stress aurait pu réduire la colonne vertébrale, le kidnappeur aurait pu pratiquer une circoncision à l'enfant. De surcroit, Christine regarderait son enfant par le prisme d'une émotion extrême et ne serait pas en mesure d'être véritablement objective. Le cœur d'une mère - dit le docteur Tarr - guidé par les émotions et l'instinct, se révolte au changement et c'est pour cela qu'elle persiste à ne pas reconnaître dans le garçon son propre fils. Pourtant, continue le docteur, cela ne change pas les données de la réalité : "Je suis prêt à mettre ma théorie à l'épreuve en toute objectivité, Mme Collins. Et vous?»

Dans la scène suivante, Christine, enragée, téléphone au capitaine Jones en lui disant que le docteur Tarr l'a emmenée faire un tour dans le quartier en la montrant comme une mère infâme, incapable de reconnaître son fils. Le lendemain un article paraît dans le Times et déshonore Christine en lui imputant de façon latente les responsabilités du dépérissement et des changements physiques de l'enfant et en insinuant que ces transformations ont eu lieu après le retour à la maison. Grâce à l'aide du pasteur de l'église presbytérienne Gustave Briegleb (John Malkovich), engagé depuis longtemps dans la dénonciation de la violence, de la corruption et de l'incompétence de la police de Los Angeles, Christine réussit à trouver des témoignages de médecins et des enseignantes de Walter lui permettant de démontrer l'erreur de la police. Ensuite, elle fait une déclaration à la presse dans laquelle elle dénonce publiquement l'action fautive des autorités. Ainsi, elle essaie d'échapper à l'image construite par la police et par les journalistes, qui commencent à la décrire comme une femme émotionnellement bouleversée, ou - encore pire - irresponsable et dépourvue de toute crédibilité. Christine, de son côté, amène des preuves objectives pour attester sa vérité et lie publiquement son image à celle d'un prêtre très connu et très apprécié dans la communauté.

24 Après une première tentative - vaine - pour remédier à l'erreur de la police en mettant en jeu sa certitude maternelle, Christine s'attèle à gérer le conflit entre les deux vérités et à résister aux attaques de la police qui continue entretemps de nier son erreur. Pour atteindre cet objectif, elle essaie de se procurer le plus grand nombre de 
témoignages pour soutenir la légitimité de ses affirmations et en même temps, elle construit une version publique de sa propre histoire.

Grâce à l'appui de la figure du prêtre, Christine réussira à persuader l'opinion publique, qui se mobilisera en sa faveur. Toutefois, sa condition sociale - Christine est une femme seule dans les États-Unis prohibitionnistes au seuil de la Grande Dépression permettra aux autorités d'exercer leur pouvoir et d'agir selon un système de valeurs qui seront défavorables à la femme.

C'est à ce moment que la réaction du capitaine Jones devient impitoyable face à l'attaque subie par son département. Il entamera un chemin orienté par une des trois procédures d'exclusion qui, selon Michel Foucault, contrôlent, sélectionnent, organisent et distribuent la production du discours à l'intérieur de chaque société afin d'éviter les pouvoirs et les dangers: l'opposition raison et folie ${ }^{20}$. La parole de Christine et les témoignages qu'elle apportera seront neutralisés par d'autres paroles, beaucoup plus puissantes et influentes, lorsqu'elle sera déclarée folle.

\section{Affidavit et hospitalisation}

Dès qu'il a pris connaissance de la mobilisation de Christine, le capitaine Jones la convoque dans son bureau pour un entretien à l'issue duquel elle sera internée :

J. J. Jones. - Vous nous avez causé beaucoup d'ennuis, Mme Collins. Cette situation est très gênante pour le service de police.

C. Collins. - Je ne voulais pas vous embarrasser.

J. J. Jones. - Non, bien sûr que non. Mais pour nous remercier d'avoir trouvé votre fils, vous dites aux journaux qu'on ne sait pas différencier deux garçons. Vous voulez rire de nous, c'est ça? Ça vous fait rire?

C. Collins. - Bien sûr que non. Je veux que vous retrouviez mon fils.

J. J. Jones. - Savez-vous quel est votre problème, Mme Collins? Vous rejetez vos responsabilités maternelles.

C. Collins. - Quoi ?

J. J. Jones. - Vous aimiez être libre, c'est ça? Vous aimiez ne pas avoir à vous occuper de votre fils. Vous aimiez aller où vous vouliez, faire ce que vous vouliez et voir qui vous vouliez. Mais on a trouvé votre fils et on l'a ramené. Et maintenant, il vous incommode. Vous avez donc inventé cette histoire pour que l'État s'en occupe et l'élève à votre place. N'est-ce pas?

C. Collins. - C'est faux!

J. J. Jones. - Ah oui ?! Même le petit dit qu'il est votre fils. Pourquoi dirait-il ça? Pourquoi?

C. Collins. - Je l'ignore, mais il ment.

J. J. Jones. - Peut-être. C'est peut-être un menteur. Mais c'est comme ça qu'il a été élevé, n'est-ce pas? Le mensonge fait partie de vos vies. Vous êtes une menteuse, une fautrice de troubles. Vous ne devriez pas être en liberté.

C. Collins. - Attendez un peu...

J. J. Jones. - Soit vous savez que vous mentez, soit vous ignorez si vous mentez ou si vous dites la vérité. Alors, qu'est-ce que vous choisissez? Êtes-vous une mère infâme? Ou simplement folle? Selon moi, c'est les deux seules options.

C. Collins. - Je n'ai pas à vous écouter.

J. J. Jones. - Vous voulez des expert? Des médecins? En voici. Infirmières [il parle aux infirmières]. Madame Collins, maintenez-vous toujours que ce garçon n'est pas votre fils?

C. Collins. - Oui. Je le soutiens toujours.

J.J. Jones [il parle aux infirmières]. - Vous allez la transférer au service de psychiatrie du Centre Hospitalier de Los Angeles. Veuillez inscrire que l'accusée 
déclare que les policiers l'ont flouée, qu'ils lui ont remis un garçon et qu'ils l'ont forcée à croire que c'était son fils. Emmenez-la! photographie qui la représente près du train avec l'enfant à peine retrouvé et la déclaration suivante où elle affirmait de ne pas être sa mère. Selon l'évaluation psychiatrique, Mme Collins est atteinte de paranoïa, de délires de persécution et de détachement de la réalité, et c'est pour ces raisons que l'on prescrit un enfermement jusqu'à qu'elle recouvre la raison - Mme Collins représentant, en l'état, un danger pour la société. mère négligente, qui a renié son fils pour ne plus s'en charger, ou bien elle est affectée par des troubles psychiatriques altérant et faussant sa perception de la réalité, et ne lui permettant dès lors plus de reconnaître son enfant. Il n'y a pas d'autres options. Ainsi, le système institutionnel se transforme et devient un système de domination, d'intimidation et d'assujettissement, qui déforme la vérité dans le but de jeter le discrédit sur la mère et, de cette façon, de compromettre sa crédibilité aux yeux de l'opinion publique, qui ne constitue pas un simple spectateur mais un élément constitutif des dynamiques discours-pouvoir ${ }^{21}$.

ipulation de l'opinion publique et agressivité verbale. Nous sommes face à une véritable attaque personnelle qui ne peut répondre aux conditions de félicité et qui ne peut se réaliser pleinement qu'à condition de rester cachée ou, mieux encore, de se faire passer pour un autre acte linguistique ${ }^{22}$ (dans le cas en question, pour une accusation fondée ou un diagnostic médical). Il s'agit d'une attaque qui nie subrepticement à la victime la réciprocité de la parole, en soustrayant ainsi celui qui porte l'attaque à la confrontation directe ${ }^{23}$. qui contient la possibilité de résistance, disposant ainsi la reconnaissance de l'autre en tant qu'élément constitutif du pouvoir même ${ }^{25}$. Dans la dissymétrie de la relation de pouvoir entre Christine et le policier, on a l'impression que la femme n'a aucune chance et qu'il ne lui reste qu'à subir passivement cet abus. À vrai dire, émerge ainsi l'image d'une femme qui lutte, qui exerce sa forme d'opposition en s'accrochant et en se défendant par la répétition à chaque fois de la même vérité. Ce faisant, Christine opte pour l'attribution discursive qui la définit et qui esquisse son portrait de mère folle. La stratégie de l'autorité, en fait, consiste, non pas en une attaque à l'égard des affirmations de Mme Collins, mais en une sorte d'« annihilation » de son discours établi par une déclaration et une attestation de folie.

ne s'agit pas d'une accusation dépourvue de tout fondement, mais d'une construction qui tire sa force de l'erreur de Christine Collins à la gare, par la contradiction entre une La déclaration de folie confère à Christine une liberté de parole totale, au prix de ne pouvoir proférer que des choses insensées. Foucault, en comptant la folie parmi les principes de partition et d'exclusion, explique que, depuis le Moyen Âge, le fou est celui dont le discours ne peut pas circuler comme celui des autres; la parole du fou est considérée comme vide et sans effet. Elle n'a ni vérité ni importance; il s'agit d'une parole qui tombe dans le néant, rejetée dès qu'elle a été proférée ${ }^{26}$. Les mots de Christine constituent le lieu d'un discrédit possible de la police ; elles contiennent en germe un pouvoir dangereux, dont les autorités ont besoin de s'emparer. Dans 
l'impossibilité de parvenir à un accord (la femme ne changera jamais son opinion car ce garçon n'est pas son enfant) et d'apparaitre violentes face à l'opinion publique, les autorités n'ont qu'une seule possibilité pour anéantir la parole de Christine : le recours à une forme de coercition ressemblant à une action légitime. En effet, à l'époque, il était possible qu'un capitaine de police ordonne l'emprisonnement ou l'enfermement d'un citoyen de Los Angeles à l'intérieur d'un institut psychiatrique sans mandat, sans expertise, et sans procès régulier. En fait, c'est réellement lors du procès entamé contre le capitaine Jones que les lois et les procédures en vigueur ont été réexaminées et changées; et, par conséquent, de nombreux emprisonnements prévus par le «Code 12 » ont été suspendus.

33 La crédibilité de la version racontée par Christine est sapée par un moyen puissant; grâce à cette asymétrie du positionnement, la déclaration de l'institution est à même d'infirmer la vérité de l'autre. Autant linguistique que violente est l'issue que l'on offre à la mère pendant sa réclusion : elle aurait la possibilité d'être libérée en échange d'une confession.

\section{La confession}

Comme nous l'avons mentionné dans le premier paragraphe, le docteur Jonathan Steele - le psychiatre dirigeant l'institut des maladies mentales - insiste sur ce qui s'est produit à la gare pour saper l'ethos de Christine et lui mettre la pression:

Dr J. Steele. - D'après votre dossier, vous croyez que la police a remplacé votre fils par un faux garçon.

C. Collins. - Non, je n'ai pas dit que c'était un faux garçon. Mais ce n'est pas mon fils. On a ramené le mauvais garçon et mon fils est toujours disparu.

J. Steele. -- C'est bizarre, car j'ai un article de journal où on voit une photo de vous, à la gare, accueillant votre fils. C'est bien vous, n'est-ce pas ?

C. Collins. - Oui.

J. Steele. - Oui.

C. Collins. - Oui.

J. Steele. - Donc, au début, c'était votre fils, mais plus maintenant. Ça fait longtemps que ça dure? Selon vous, les gens changent ou deviennent autre chose?

C. Collins. - Les gens ne changent pas.

J. Steele. - Vous croyez que les gens ne changent pas?

C. Collins. - Non, c'est ne pas...

J. Steele. - Shhh!

Cette conversation se prolonge jusqu'au moment où le docteur Steele lui pose la question de savoir si elle rencontre souvent des problèmes à distinguer la réalité de la fantaisie. La calomnie du médecin consiste à accuser Mme Collins de ne pas posséder cette aptitude de discernement, en ne lui laissant que deux alternatives : admettre sa maladie et se soumettre au traitement médical ou bien démontrer sa bonne santé mentale en signant une déclaration, c'est-à-dire une confession, dans laquelle elle affirme s'être trompée, en niant ce qu'elle avait précédemment dit et disculpant ainsi la police.

C. Collins. - Je ne veux pas avaler un médicament inconnu. Je ne suis pas folle. Je n'ai pas besoin de ça.

J. Steele. - Vous n'êtes pas folle.

C. Collins. - Je ne suis pas folle.

J. Steele. - Non. Vous êtes saine d'esprit.

C. Collins. - Oui, c'est vrai. 
J. Steele. - Donc, vous n'hésiterez pas à signer ceci. En signant ça, vous attestez que vous aviez tort de dire que le garçon que la police a ramené n'était pas votre fils. Vous attestez aussi que la police a eu raison de vous envoyer ici en observation et vous la dégagez de toute responsabilité. Signez ici.

C. Collins. - Je ne signe pas ça. Je refuse de signer.

J. Steele. - Votre état ne s'est pas amélioré, alors. Si vous le signez, vous pourrez partir demain matin.

C. Collins. - Mais je n'avais pas tort. Ce n'est pas mon fils. Il est toujours disparu.

J. Steele. - Madame Collins, vous êtes agitée.

C. Collins. - Oui, mais... Je refuse de signer ça, car ce n'est pas mon fils... Ce n'est pas mon fils. Mon fils est toujours disparu!

J. Steele. - Infirmier ! La patiente est hystérique. Donnez-lui des sédatifs.

transformera dans la deuxième partie du film en une possibilité de réhabilitation : sa persévérance lui rendra possible une libération soudaine. Alors que Christine est enfermée dans l'institut pour malades mentaux, la police a arrêté Gordon Northcott - un meurtrier d'une vingtaine d'enfants, parmi lesquels figure justement Walter Collins. Ayant appris cette nouvelle par le pasteur Briegleb qui lui montrera la page du journal, le docteur Steele libère la patiente. Cependant, avant de la libérer, il lui pose à nouveau la question de savoir si elle est disposée à changer ou non sa version de l'histoire :

J. Steele. - Madame Collins, pour la dernière fois : allez-vous signer cette lettre ou

non?

C. Collins. - Non.

J. Steele. - Vous pouvez partir. Vous êtes libre.

Jusqu'à ce moment du film, la vérité était déguisée et remplacée par une autre. Une fois manifestée et rétablie, elle permettra aux conséquences filmiques et juridiques s'appuyant sur elle de (re)démarrer. Les deux procès, dont parlera la deuxième partie du long-métrage, même s'ils ne rendront pas son fils à Christine, dénonceront au moins les abus subis.

\section{Conclusions}

Confiance et mensonge caractérisent tout ordre social. La confiance est un élément précontractuel de la vie sociale, partie constitutive du rapport de l'enfant avec la mère, un ciment à la base des rapports humains dans la variété de leurs formes, une composante essentielle du "capital social ${ }^{28}$ ». Elle est toujours liée à une attente, ou mieux à une sorte de prédiction du comportement d'autrui ; d'où sa relation forte avec la vérité et le fait de dire la vérité. Elle est en relation étroite avec le fait de tenir la parole donnée au cours du temps et lors des actions, avec la possibilité toujours présente du mensonge, de l'erreur d'évaluation, de la manipulation, de l'ignorance et de l'incertitude qui en découle. 
La confiance n'est pas d'ordre exclusivement anthropologique, mais se transforme sur la base des diversités historico-sociales et locales: tous les hommes ont par nature tendance à faire confiance à l'autre, à croire en ce qu'on apprend par les maîtres ou par les médecins. Cependant, dans la modernité, la confiance a assumé un rôle particulier et prépondérant de manière à ce que, en suivant une expression de Georges Simmel, la vie moderne est une économie de crédit, bien au-delà du sens strictement économique du terme ${ }^{29}$. Selon Simmel, plus la vie culturelle devient riche et épanouie, plus elle repose sur des conditions préalables que l'individu ne peut pas étudier ni vérifier jusque dans leur fondement, mais qu'il doit accepter par confiance, en gardant foi dans l'honnêteté de l'autre ${ }^{30}$. À l'époque moderne :

Nous fondons nos décisions les plus importantes sur un système complexe de représentations dont la plupart supposent la certitude de ne pas être trompés. C'est pourquoi, dans la vie moderne, le mensonge fait beaucoup plus de ravages, il met en question les fondements de l'existence beaucoup plus que ce n'était le cas autrefois ${ }^{31}$.

41 Au fur et à mesure que le poids du mensonge change, que le climat et les valeurs de référence où s'instituent les rapports de confiance se métamorphosent, les rapports changent entre discours, vérité et pouvoir (objet spécifique de notre analyse). Plus une société s'amplifie, plus les informations augmentent, ainsi que les possibilités de les partager et d'y avoir accès, plus il devient difficile et compliqué d'apprendre à faire confiance de manière appropriée. Évidemment, il ne s'agit pas de donner des instructions pour démasquer les abus du pouvoir et les ruses; ni même d'élaborer des théories abstraites de l'argumentation linguistique qui puissent les définir de façon normative afin de s'en défendre. Au contraire, la stratégie que je propose ici vise à analyser un discours concret, car je suis persuadée que pour comprendre ce qui nous lie à la vérité de nos affirmations et en même temps pour nous défendre contre des formes dangereuses d'attaque verbale, une aide véritable ne peut venir que de la spécificité des contextes et des cas singuliers - passés au crible d'un exercice critique réitéré. Décrire des formes de violence verbale nous aide à voir comment exercer la résistance à la calomnie, c'est-à-dire le savoir-faire pratique qu'on peut tirer de notre réflexion.

Nous avons examiné, au sein du film Changeling de Clint Eastwood, deux macrocatégories de phénomènes langagiers: une qui concerne les éléments discursifs par lesquels nous sommes enclins à accorder notre confiance; l'autre se référant aux stratégies utilisées pour résister à la méfiance et au discrédit, ainsi que pour faire face aux accusations d'avoir donné des déclarations fausses ou insensées.

Depuis l'Antiquité, la rhétorique a reconnu que la relation entre les sujets parlants - la fides - est en mesure de renforcer les mots, en laissant implicite le fait que le rapport entre subjectivité et discours ne permet aucune scission entre celui qui parle ou qui écoute et ce qui est dit. On ne peut pas séparer les discours des rôles sociaux et des relations de pouvoir qui renforcent, affaiblissent et parfois même annulent, la puissance des mots et la crédibilité de l'énonciateur. Dans le cas présent, on peut observer dans quelle mesure la crédibilité de l'orateur était conditionnée par le système de valeurs partagées par la société. Ces valeurs émergeant au niveau discursif réalisaient un écart remarquable entre l'image véhiculée par les discours d'un capitaine de police et celle d'une mère célibataire dans la société nord-américaine des années 1920. Dans la lignée d'une enquête qui lie les aspects techniques et ceux non techniques de l'ethos, nous avons montré que l'ethos du capitaine tire sa force, tout au long du film, de l'acte linguistique du serment et de la façon dont il se représente: son portrait est 
rendu possible et efficace par l'appui de son rôle institutionnel. L'ethos de Christine Collins - se révélant beaucoup plus intéressant d'un point de vue théorique parce que cet ethos est mis à l'épreuve par la résistance face à la diffamation des autorités - est manifestement plus faible et fragile. La vérité est écrasée par le préjugé, les témoignages objectifs sont toujours détournés, les sentiments de préoccupation maternelle sont transformés en symptômes d'hystérie, l'intérêt passionné pour sa propre cause est déformé en absence de rationalité.

Ce que nous savons d'un individu contribue à définir la situation et les attentes; mais cela ne veut nullement dire que dans cette perspective de la représentation théâtrale ${ }^{32}$ le sujet doit rester écrasé par cette image. Le long-métrage montre en effet la possibilité de retravailler ${ }^{33}$ son propre ethos, de déterminer à chaque fois des stratégies de résistance et d'opposition à la tyrannie du discours du pouvoir. Les conditions éthiques de la vérité ne sont pas réductibles à des règles formelles : à chaque fois, celui qui parle a la possibilité d'exprimer le courage de lier son existence à la prise de parole, en acceptant les implications politiques, sociales et personnelles de la vérité qu'il affirme. Ainsi, même celui qui écoute a la liberté et le devoir d'accueillir ou de rejeter la parole de l'autre en tant que parole véritable, de mettre à l'épreuve les impressions issues du discours de l'autre et d'évaluer de façon critique le système de croyances implicites et sous-jacentes.

\section{NOTES}

1. E. Goffman, The Presentation of Self in Everyday Life, Garden City, N. Y., Doubleday, 1959, p. 2 ; La mise en scène de la vie quotidienne, tome 1 , La Présentation de soi, trad. A. Accardo, Paris, Éditions de Minuit, 1973, p. 12 (suite de la citation : « les autres lui font normalement crédit et lui offrent pendant qu'il est en leur présence la contrepartie d'une chose dont ils ne pourront établir la véritable valeur qu'après qu'il les aura quittés »).

2. The Changeling (titre français: L'Échange), 2008. Réalisation: Clint Eastwood. Scénario : J. Michael Straczynski. Photographie: Tom Stern. Montage : Joel Cox, Gary D. Roach. Musique: Clint Eastwood. Décors : James J. Murakami. Acteurs principaux: Angelina Jolie (Christine Collins), John Malkovich (révérend Gustav Briegleb), Jeffrey Donovan (Capitaine J.J. Jones), Micheal Kelly (Lieutenant Lester Ybarra), Colm Feore (le chef de la police, James E. Davis), Jason Butler Harner (Gordon Northcott), Amy Ryan (Carol Dexter), Devon Conti (Arthur Hutchins), Gattlin Griffith (Walter Collins), Denis O'Hare (le docteur Jonathan Steele), Peter Gerety (le docteur Earl W. Tarr). Production: Clint Eastwood, Brian Grazer, Ron Howard et Robert Lorenz. Sociétés de production : Malpaso Production, Imagine Entertaintment et Relativity Media. Société de distribution : Universal Pictures. Pays d'origine : États-Unis. Langue originale : anglais. Durée : 141 minutes. Date de sortie : 20 mai 2008 (Festival de Cannes).

3. G. Agamben, Il sacramento del linguaggio. Archeologia del giuramento, Rome-Bari, Laterza, 2008 ; É. Benveniste, «L'expression du serment dans la Grèce ancienne ", Revue de l'histoire des religions, 1948, p. 81-94 ; id., Le vocabulaire des institutions indo-européennes, Paris, Les Éditions de Minuit, 1969, vol. 2, livre 1, chap. 3, "Ius et le serment à Rome », p. 111-122, et chap. 8, «Le serment en 
Grèce », p. 163-175 ; P. Prodi, Il sacramento del potere. Il giuramento politico nella storia costituzionale dell'Occidente, Bologne, Il Mulino, 1992.

4. Le terme latin fides désigne aussi bien la confiance que l'on peut avoir en quelqu'un, que celle que l'orateur suscite chez l'auditeur, sa crédibilité et le crédit dont il profite. La fides est donc à la fois quelque chose que l'on peut avoir et donner : j'ai de la confiance en quelqu'un et quelqu'un en même temps me fait confiance (É. Benveniste, Le vocabulaire, op. cit., p. 115-121).

5. J. Austin, How to Do Things with Words, Oxford-New York, Oxford University Press, 1962.

6. "Or je jure est une forme de valeur singulière, en ce qu'elle place sur celui qui s'énonce je la réalité du serment. Cette énonciation est un accomplissement : “jurer" consiste précisément en l'énonciation je jure par quoi Ego est lié. L'énonciation je jure est l'acte même qui m'engage, non la description de l'acte que j'accomplis » (É. Benveniste, « De la subjectivité dans le langage » dans Problèmes de linguistique générale, Paris, Gallimard, 1966, p. 265).

7. G. Agamben, Il sacramento, op. cit., p. 95.

8. J. Searle, Speech Acts: An Essay in the Philosophy of Language, Cambridge, Cambridge University Press, 1969. Cependant, Searle ne fait référence qu'à la promesse, sans mentionner le serment.

9. É. Benveniste, Le vocabulaire, op. cit., p. 164.

10. Id., "L'expression... ", op. cit., p. 82.

11. G. Simmel, Secret et sociétés secrètes, Strasbourg, Circé, 1908 [1991].

12. F. Piazza, La Retorica di Aristotele. Introduzione alla lettura, Rome, Carocci, 2008, p. 16-17; Benveniste É., Le vocabulaire, op. cit., p. 103-121 ; G. Agamben, Il tempo che resta. Un commento alla "Lettera ai romani », Turin, Bollati Boringhieri, 2000, p. 106-127 (Sesta giornata), dont p. 106-108 sur le giuramento (Le Temps qui reste. Un commentaire de l'Épître aux Romains, Paris, Rivages, 2004, ouvrage divisé en « six journées »).

13. Elles se distinguent des preuves non techniques (atechnoi) ainsi définies car elles se trouvent déjà disponibles, tels que les témoignages, les confessions, les documents écrits, etc. Au contraire, les preuves techniques sont toujours à inventer par l'orateur grâce à une méthode ou technique rhétorique (Aristote, Rhét., 1355b35-39). Pour une analyse de la Rhétorique d'Aristote, voir F. Piazza, La Retorica, op. cit.

14. Aristote, Rhét., 1356a.

15. Ibid., 1356a4-13; F. Piazza, La Retorica, op.cit., p.91-92; S. Di Piazza, «Fiducia e argomentazione. Una prospettiva aristotelica », Rivista italiana di filosofia del linguaggio, $\mathrm{n}^{\circ} 6,3$, 2012, p. 41-52.

16. Aristote, Rhét., 1356a4-13.

17. Le débat sur les éléments contribuant à l'image de soi que l'orateur produit, telle qu'elle a été conçue dans la Grèce classique, se poursuit aujourd'hui. Pour une reconstruction du débat dans l'Antiquité, voir F. Woerther, L'èthos aristotélicien. Genèse d'une notion rhétorique, Paris, Vrin, 2007 ; R. Amossy, L'argumentation dans le discours, Paris, Colin, 2000 [2010], p. 63-64; La présentation de soi. Ethos et identité verbale, Paris, Presses Universitaires de France, p. 19. Pour la notion contemporaine d'ethos, je renvoie à R. Amossy éd., Images de soi dans le discours. La construction de l'ethos, Lausanne-Paris, Delachaux et Niestlé, 1999 et R. M. Zagarella, «Ispirare fiducia : l'ethos nella retorica contemporanea ", Blityri. Studi di storia delle idee sui segni e le lingue, Pise, Edizioni ETS, 2015 (à paraître).

18. C. Plantin, Les bonnes raisons des émotions. Principes et méthode pour l'étude du discours émotionné, Berne, Peter Lang SA, 2011, p. 34-36.

19. C. Perelman et L. Olbrechts-Tyteca, Traité de l'argumentation. La nouvelle rhétorique, Paris, Presses Universitaires de France, 1958, § 69.

20. M. Foucault, L'ordre du discours, Paris, Éditions Gallimard, 1971.

21. M. Foucault., "The Subject and the Power" dans H. Dreyfus et P. Rabinow éd., Michel Foucault : Beyond Structuralism and Hermeneutics, Chicago, University of Chicago Press, 1982. 
22. R. Pomelli, «Triangolazione diabolica e terzietà nella grecia antica », Rivista italiana di Filosofia del linguaggio, $\mathrm{n}^{\circ}$ 6, 3, 2012, p. 95-107 ; F. Piazza, «Calunnie, diavoli e macchine del fango. Sulla nozione di diabole nella retorica greca ", Blityri. Studi di storia delle idee sui segni e le lingue, op. cit.

23. R. Pomelli, Triangolazione, op. cit.; N. Loraux, La cité divisée. L'oubli dans la mémoire d'Athènes, Paris, Éditions Payot \& Rivages, 1997.

24. Pour Foucault, ce modèle est fort inadéquat, car il pose d'une part le pouvoir législatif et d'autre part un sujet obéissant: "D'abord parce que ce serait un pouvoir pauvre dans ses ressources, économe de ses procédés, monotone dans les tactiques qu'il utilise, incapable d'invention et comme condamné à se répéter toujours lui-même. Ensuite parce que c'est un pouvoir qui n'aurait guère que la puissance du "non"; hors d'état de rien produire, apte seulement à poser des limites, il serait essentiellement anti-énergie; tel serait le paradoxe de son efficacité : ne rien pouvoir, sinon faire que ce qu'il soumet ne puisse rien à son tour, sinon ce qu'il lui laisse faire. Enfin parce que c'est un pouvoir dont le modèle serait essentiellement juridique, centré sur le seul énoncé de la loi et le seul fonctionnement de l'interdit. Tous les modes de domination, de soumission, d'assujettissement se ramèneraient finalement à l'effet d'obéissance. » (M. Foucault, La Volonté de savoir. Histoire de la sexualité 1, Paris, Gallimard, 1976, p. 112-133).

25. Comme l'explique Michel Foucault (dans The Subject, op.cit., p. 208-226): «[...] A power relationship can only be articulated on the basis of two elements which are each indispensable if it is really to be a power relationship: that "the other" (the one over whom power is exercised) be thoroughly recognized and maintained to the very end as a person who acts; and that, faced with a relationship of power, a whole held of responses, reactions, results, and possible inventions may open up. »

26. M. Foucault, L'ordre, op. cit.

27. M. Foucault, Mal faire, dire vrai. Fonction de l'aveu en justice. Cours de Louvain, Louvain-Chicago, Presses Universitaires de Louvain/University of Chicago Press, 1981 [2012].

28. «Social capital»: R. D. Putnam, Bowling Alone: The Collapse and Revival of American Community, New York, Simon \& Schuster, 2000.

29. G. Simmel, Secret, op. cit., p. 16.

30. Ibid.

31. Ibid.

32. E. Goffman, The Presentation, op. cit.

33. R. Amossy, La présentation, op. cit.

\section{AUTEUR}

\section{ROBERTA MARTINA ZAGARELLA}

Academia Belgica / CNR-ITB 